\section{The prevalence of self-reported smoking and validation with urinary cotinine among commercial drivers in major parks in Lagos, Nigeria}

\author{
Obianuju B. Ozoh, ${ }^{1}$ Michelle G. Dania, ${ }^{1}$ \\ Elvis M. Irusen² \\ 1Department of Medicine, College of \\ Medicine, University of Lagos, Nigeria; \\ ${ }^{2}$ Division of Pulmonology, University of \\ Stellenbosch, Cape Town, South Africa
}

\section{Abstract}

The validity of self-reported smoking is questionable because smokers are inclined to deny smoking. We aimed to determine the prevalence of self-reported smoking among intra-city commercial drivers in Lagos, and assess its validity based on urinary cotinine assessment. This study was conducted at three major motor parks in Lagos, Nigeria. Information on smoking status and habits was obtained from 500 consecutive male drivers using a structured questionnaire during a face-to-face interview. Eighty-one self-reported smokers and non-smokers were selected by systematic random sampling for urinary cotinine assessment using cotinine strips. The prevalence of self-reported smoking was compared to the prevalence of smoking based on urinary cotinine and the specificity and positive predictive values of self-reported smoking was determined. Prevalence of self-reported current smoking was $32 \%$ and $17.9 \%$ of nonsmokers were passive smokers. Among 81 drivers in whom urinary cotinine assessment was performed, the prevalence of smoking based on self-report was 34 (42\%) compared to 41 (50.6\%) when based on urinary cotinine, $\left(\mathrm{X}^{2}=38.56, \mathrm{P}<0.001\right)$. The rate of misclassification among self-reported non-smokers as smokers was $21.3 \%$ and misclassification rate for self-reported smokers as non-smokers was $8.8 \%$. The sensitivity of self-reported smoking in accurately classifying smoking status was $91.2 \%$ and the specificity was $78.7 \%$. The prevalence of self-reported cigarette smoking among commercial drivers in Lagos is high and a significant proportion of self-reported non-smokers are passive smokers. Self-reported smoking status obtained during face-to-face interview appears unreliable in obtaining accurate smoking data in our locality.

\section{Introduction}

Cigarette smoking is a growing problem in developing countries and about $80 \%$ of deaths attributable to tobacco are expected to occur in this region by $2030 .{ }^{1}$ According to the World Health Organization World Mental Health Survey (2002 to 2003), 16.8\% of Nigerians use tobacco (cigarettes, cigars or pipe), however the prevalence is much higher among certain population groups such as commercial drivers in whom rates range from $25 \%$ to $85 \%{ }^{2-5}$

Self-reported smoking is generally used to determine smoking prevalence in the population. However, the validity of self-reported smoking is often questionable because smokers are inclined to underestimate the amount smoked or deny smoking altogether.,7 Smokers deny smoking because of social or medical disapproval, misunderstanding, intentional deception, embarrassment, denial, and shame. ${ }^{6,7}$ The percentage of subjects who deny smoking ranges from $1.4 \%$ in broad based epidemiologic studies and up to $35 \%$ among pregnant women. ${ }^{8}$ To validate the prevalence of self-reported smoking, certain biomarkers of cigarette smoke such as nicotine, cotinine, thiocyanate, carboxylated hemoglobin and exhaled breath carbon monoxide can be used. ${ }^{9,10}$ Cotinine, the major metabolite of nicotine, specific for tobacco is currently considered the best indicator of cigarette exposure (active and passive smoking) and has a greater sensitivity and specificity than other biomarkers. ${ }^{9}$ It has a long half-life (10-20 h) that allows for detection of recent smoking for up to four to seven days (compared to about two hours for nicotine) after the last episode of smoking. Cotinine can be measured in various biological fluids including plasma, urine, saliva, breast milk and cervical mucus. Measurement can be performed using either chromatographic techniques or by immunoassay analysis and results can be obtained quantitatively by laboratory estimations or qualitatively by use of cotinine test strips. ${ }^{8,10-13}$ Use of cotinine test strip is simple, less costly than the laboratory tests, provides results in minutes and is a valid method for confirming self-reported smoking. ${ }^{14}$ Specifically urinary cotinine strips have been demonstrated to compare favorably with the gold standard the gas chromatography and mass spectrometry laboratory assay. ${ }^{14,15}$

Accurate assessment of smoking status is important in generating regional and national estimates which in turn guide the allocation of resources and the setting of health priorities. Smoking prevalence data in Nigeria have largely relied on the self-reported smoking status and therefore may be subject to bias. There is a need to validate the utility of self-report as a means of determining smoking prevalence in
Correspondence: Obianuju B. Ozoh, Department of Medicine, College of Medicine, University of Lagos, PMB 12003, Lagos, Nigeria.

E-mail: ujuozoh@yahoo.com; oozoh@unilag. edu.ng

Acknowledgements: we wish to acknowledge our research assistant Mr Albert Jegede for his role in gathering data for this study.

Key words: prevalence, smoking, drivers, Nigeria cotinine.

Contributions: OB0, study conceptualization and design, data acquisition and analysis, manuscript drafting and final revision for intellectual content; MGD, study conceptualization and design, data acquisition, manuscript final revision for intellectual content; EMI, study conceptualization and design, data analysis and manuscript final revision for intellectual content.

Conflict of interests: the authors declare no potential conflict of interests.

Received for publication: 3 January 2013.

Revision received: 23 December 2013.

Accepted for publication: 4 February 2014.

This work is licensed under a Creative Commons Attribution NonCommercial 3.0 License (CC BYNC 3.0).

(C) Copyright O.B. Ozoh et al., 2014

Licensee PAGEPress, Italy

Journal of Public Health in Africa 2014; 5:316

doi:10.4081/jphia.2014.316

the Nigerian context. We therefore aimed to determine the prevalence of self-reported smoking among commercial drivers in the Lagos metropolis and the validity of this using urinary cotinine assessment.

\section{Materials and Methods}

This was a cross-sectional study of consecutively consenting intra-city commercial bus and taxi drivers in the Lagos metropolis. Ethical approval was obtained from the Health Research Ethics committee of the Lagos University Teaching Hospital Idi-Araba, Lagos (ADM/DCST/HREC/310). The study was conducted at the three major commercial motor parks in Lagos Mainland, situated at Ojuelegba, Idi-araba and Lawanson. These parks were selected based on information obtained from local government authorities and the National Union of Road Transport Workers (NURTW) on the ranking of the commercial motor parks based on membership of registered operators. A total of 610 commercial drivers were registered under the NURTW 
(mandatory membership required of all commercial drivers) at the 3 parks at the time of the study between September and December 2011. Verbal permission was obtained from the NURTW executive at each park, while individual written consent was obtained from each participating driver.

Data was collected from consecutively consenting drivers during the weekly NURTW meetings held at the park. Based on an initial pilot survey in which a response rate of $80 \%$ was obtained we estimated a total sample of 488 ( $80 \%$ of 610$)$ with the intention to round this up to a final sample size of 500 .

Data collection was carried out by a trained interviewer during a face-to-face encounter. Information obtained included demographic data (age gender), history of cigarette smoking and smoking habits (including the quantity and type of tobacco smoked).

We defined ever smoking as smoking at least one stick of cigarette per day for at least one year or more than 20 packs of cigarette in a lifetime and current smoking as smoking at least one puff of cigarette in the preceding 30 days. We also defined recent smoking as smoking at least one puff of cigarette in the preceding three days. History of second hand tobacco exposure (passive smoking) described as being in the presence of someone who was smoking cigarettes in the preceding three days. Use of nicotine replacement was described as use of nicotine gum, patch or nasal spray in the preceding 30 days.

Smoking dependence was assessed using the Modified Fagerström Test for Nicotine Dependence. ${ }^{16}$ This is a validated 6 item questionnaire that asks about time of first cigarette smoking in a day, smoking in forbidden places, most difficult cigarette to give up, number of sticks of cigarette smoked per day, smoking the highest quantity of cigarette in the first hour after waking and smoking when quite ill. Each question has options that are weighted one to three with a maximum total score of ten. A total score of 0-4 shows mild nicotine dependence, 5-6 medium dependence and 7-10 high nicotine dependence. ${ }^{16}$

To limit costs, urine samples were collected by systematic random sampling in a clean universal bottle from every fifth person who was a current smoker and admitted to smoking cigarette in the preceding 3 days and also from every sixth person who was not a current smoker on self-report. (An average prevalence rate of current smoking of about $30 \%$ was used based on previous studies). ${ }^{3-5}$ Urinary cotinine assessment was performed at the site of urine collection using a cotinine test strip by a trained technician following manufacturer's instructions.

The COT® one step cotinine test device DN: 1150311801 (distributed by Innovacon, Inc., San Diego, CA, USA) was used for urinary coti- nine assessment. The COT® one-step cotinine test device is a lateral flow chromatographic immunoassay for detection of cotinine in human urine at a cut off concentration of 200 $\mathrm{ng} / \mathrm{mL}$ based on the principle of competitive binding. During testing, urine specimens migrate upwards by capillary action. Cotinine, if present in the urine below $200 \mathrm{ng} / \mathrm{mL}$, does not saturate the binding sites of the antibody coated particles in the test device, the antibody coated particles is then captured by immobilized cotinine conjugate and a visible colored line appears in the test line region. If the cotinine levels exceed $200 \mathrm{ng} / \mathrm{mL}$, the colored line does not appear in the test line region but appears in the cotinine line region because it saturates all the binding sites of anti-cotinine antibodies. To serve as procedural control a line always appears at the control line region indicating that an adequate volume of specimen has been added and membrane wicking has occurred. Therefore a negative test is indicated by the appearance of two lines on the test strip (cotinine line and test line), a positive test by the appearance of one line (cotinine line) and an invalid test by the appearance of one line in the test region only.

Data was analyzed using the Statistical Software for Social Sciences version 20.0. Continuous variables were expressed as means and standard deviations. Categorical variables were expressed as frequencies and compared using the chi-square test. A P value of $<0.05$ was considered significant. The sensitivity and specificity as well as the negative and positive predictive values were determined by standard methods.

\section{Results}

A total of 500 commercial drivers participated in the study and all were male. Three hundred and eighty six (77.2\%) were married, 112 (22.4\%) were single and $2(0.4 \%)$ were separated. The age range was $20-73$ years with a mean age (years) \pm standard deviation of $42.36 \pm 11.17$. Table 1 summarizes the age distribution of the participants.

\section{Prevalence of self-reported smoking}

There were 286 (57.2\%) ever smokers that included 160 (32\%) current smokers, 214 (42.8\%) of the participants were never smokers. All the participants smoked cigarettes only and they were no pipe or cigar smokers. One hundred and thirty five (27\%) smoked cigarettes daily, 26 (5.2\%) were occasional smokers and 159 (31.8\%) were recent smokers (had smoked at least one cigarette in the preceding 3 days). They were 43 drivers (17.9\% of nonsmokers) who were passive smokers. No driver was using nicotine replacement therapy in
Table 1. Age distribution and smoking habits of study participants.

\begin{tabular}{lc}
\hline Characteristics & Frequency (\%) \\
Age distribution (in years) & \\
$15-39$ & $213(42.6 \%)$ \\
$40-65$ & $285(57 \%)$ \\
$>65$ & $2(0.4 \%)$ \\
Smoking habits & \\
Current smokers & $160(32 \%)$ \\
$\leq 10$ sticks/day & $79(49.4 \%)$ \\
$11-20$ stick/day & $58(36.3 \%)$ \\
$21-30$ sticks/day & $17(10.6 \%)$ \\
$\geq 31$ sticks/day & $6(3.7 \%)$ \\
\hline
\end{tabular}

Table 2. Categories of drivers based on self-reported smoking status and urinary cotinine assessment.

\begin{tabular}{lc} 
Category & Frequency (\%) \\
$\begin{array}{l}\text { Self-reported smokers with } \\
\text { positive urinary cotinine }\end{array}$ & $31(38.3 \%)$ \\
$\begin{array}{l}\text { Self-reported non-smokers with } \\
\text { negative urinary cotinine }\end{array}$ & $37(45.7 \%)$ \\
$\begin{array}{l}\text { Self-reported non-smokers with } \\
\text { positive urinary cotinine }\end{array}$ & $10(12.3 \%)$ \\
$\begin{array}{l}\text { Self-reported smokers with } \\
\text { negative urinary cotinine }\end{array}$ & $3(3.7 \%)$ \\
\hline
\end{tabular}

Urinary cotinine was measured in 81 drivers (randomly selected). Number of self-reported smokers amongst drivers in whom urinary cotinine was measured was $34 / 81$

any form. Table 1 summarizes the average number of cigarettes smoked per day by the current smokers. Based on their score on the Modified Fagerström Test for nicotine dependence, $131(81.9 \%)$ of the current smokers had low nicotine dependence, 22 (13.8\%) medium nicotine dependence and 7 (4.3\%) high nicotine dependence.

\section{Validity of self-reported smoking based on urinary cotinine}

Urinary cotinine assessment was carried out for 81 drivers. Prevalence of self-reported recent smoking was 34 (42\%) but urinary cotinine assessment was positive ( $>200 \mathrm{ng} / \mathrm{mL})$ in $41(50.6 \%)$. There was a statistically significant difference in the prevalence of recent smoking based on self-report compared to the prevalence based on urinary cotinine assessment $\left(\mathrm{X}^{2}=38.56, \mathrm{P}<0.001\right)$.

Thirty-one (91.2\%) self-reported smokers had positive urinary cotinine (true positives). The prevalence of positive urinary cotinine among self-reported non-smokers (false positives) was $10(21.3 \%)$ and 3 (8.8\%) self-reported smokers were classified as non-smokers based on urinary cotinine. Table 2 shows the different categories of drivers based on their self-reported smoking status in relation to positive urinary cotinine assessment. The sensitivity of self-reported smoking accurately identifying recent smokers was $91.2 \%$ and the 
specificity was $78.7 \%$ with a positive predictive value of $75.6 \%$ and a negative predictive value of $92.5 \%$.

Twenty-two (46.8\%) of the self-reported non-smokers who had urinary cotinine assessment were passive smokers and five passive smokers had positive urinary cotinine assessment. Therefore of the 10 self-reported nonsmoking drivers with positive urinary cotinine, passive smoking may have been a confounder in $5(10.6 \%)$ participants.

\section{Discussion}

Social acceptability bias affects the validity of self-reported smoking and therefore determines the reliability of smoking data obtained by this method. In this study, the prevalence of self-reported current smoking (32\%) among commercial drivers is high and a significant proportion of self-reported non-smokers are passive smokers. Among the drivers in whom urinary cotinine assessment was performed, the prevalence of self-reported smoking was lower than the prevalence of smoking based on urinary cotinine assessment. This resulted in a misclassification rate of $21.3 \%$ among selfreported non-smokers (classified as smokers based on positive urinary cotinine). For selfreported smokers, rate of misclassification as non-smokers based on a negative urinary cotinine assessment was $8.8 \%$. Self-reported smoking had a low specificity and positive predictive value in accurately determining smoking status.

The prevalence of self-reported smoking in our study is similar to that reported in other cohorts of commercial drivers in Nigeria but much higher than that in the general population. $^{2-5}$ The high prevalence of smoking among commercial drivers implies that this group are particularly vulnerable to initiating and sustaining a smoking habit. Factors such as peer pressure, availability, accessibility and affordability of cigarettes, as well as high stress levels associated with the job and the perceived need for stimulants use may contribute to the high prevalence of smoking among commercial drivers. ${ }^{4-5,17}$ Commercial drivers are thus an important target group in controlling tobacco use in Nigeria. The significant proportion of light smokers with low nicotine dependence in our study implies that despite the high smoking prevalence, tobacco cessation and control programs are likely to be effective in this group. Furthermore, the substantial proportion of passive smokers found in our study signifies a low level of knowledge among the non-smoking drivers of the dangers of exposure to second hand cigarette smoke and hence failure to protect themselves from their smoking counterparts. This demands an intensive education program for commercial drivers that highlights the harmful effects of cigarette smoking including passive smoking so that non-smoking drivers can take adequate precautions. Cigarette smokers have approximately a 20 fold increase in lung cancer risk compared to never smokers and passive smoking increases the risk of lung cancer by $20-25 \%{ }^{18,19}$ The Tobacco Control Act which regulates the advertising, and sale of cigarettes and prevents exposure of non-smokers to cigarette smoke by restricting smoking in public areas is yet to be signed into law in Nigeria and individuals must therefore make personal efforts to avoid exposure to second-hand smoke. Tobacco companies have also found a haven in developing countries such as ours where the Tobacco Control Act is not fully implemented for tobacco manufacturing, advertising and inappropriate sales which increases the availability, affordability and use of cigarettes. For instance, cigarettes are freely sold (per stick) in most commercial motor parks in Nigeria.

The high rate of misclassification of nonsmokers as current smokers based on positive urinary cotinine suggests that some drivers deliberately falsified their smoking status. Self-reported smoking was therefore not reliable in determining smoking prevalence among our study cohort. The unreliability of self-reported smoking as a means of determining smoking status has been described among various populations. A systematic review of about 67 studies in adults showed a trend of underestimation of smoking status when based on self-report compared to cotinine assessment. ${ }^{7}$ The misclassification rate among self-reported non-smokers appears more marked among populations that are attending the hospital for conditions such as pregnancy (35\%), bronchoscopy clinic (18\%) and lung cancer screening (7\%) where the information was obtained during face to face interviews compared tostudies in which information was obtained from self-administered questionnaire during regular screening exercises or from general population surveys. ${ }^{12,20-24}$ On the other hand, the National Health and Nutrition Examination Survey (NHANES) a general population survey and a community based survey in Finland, found self-reported smoking to be quite reliable in determining smoking prevalence. Also, among educated young adults, self-reported smoking also appeared reliable. ${ }^{25}$ This therefore suggests that the reliability of self-reported smoking in determining smoking prevalence varies among various populations and depends on various factors such as the means of administering the questionnaire, the level of education of the respondents and the setting in which the information was obtained. To our knowledge, no earlier study has validated the reliability of self-reported smoking in Nigeria, and our findings are quite significant as the misclassification rate we found is higher than in other populations. This implies that certain factors such as cultural disapproval for smoking and low level of education among the drivers may have contributed to the inaccurate self-reporting of their smoking status.

The cut off level for positive urinary cotinine assessment used in this study ( $>200 \mathrm{ng} / \mathrm{mL}$ ) is not expected to identify moderate passive smokers (levels of urinary cotinine in moderate passive smokers and very light smokers usually 11-30 ng/mL) suggesting that some of the self-reported passive smokers may be current smokers who deliberately falsified their smoking status. However, if we assume that these drivers accurately reported their smoking status as passive smokers, then reasons for the positive urinary cotinine in passive smokers may include genetic variability in the coding of liver enzymes for which Africans have been shown to have higher cotinine levels compared to Caucasians. ${ }^{26}$ Other factors that reduce the enzymatic metabolism of cotinine which may also play a role in increasing cotinine positivity in light smokers and passive smokers include use of menthol cigarettes, higher lean body mass and fewer years of alcohol use. ${ }^{27}$

The misclassification rate among selfreported current smokers as non-smokers in our study was also noted and may be as a result of genetic polymorphism that occurs in certain individuals. For instance, individuals with genetic homozygous deletion of cytochrome P450 (CYP) $2 A 6$ gene (which converts nicotine to cotinine) have decreased cotinine excretion despite smoking. ${ }^{28}$

A recognized limitation in this study is the inability to perform urinary cotinine assessment for all drivers; this was as a result of the high cost of the cotinine test strips (this was a non-funded research). However, the systematic random sampling used for selection of those tested we believe reduced this potential bias.

In conclusion, the prevalence of cigarettes smoking among intra-city commercial drivers in Nigeria is high and a significant proportion of non-smokers are exposed to second-hand smoke. Our study suggests that self-reported smoking may not be a reliable means of determining smoking prevalence in our population. Further studies validating self-reported smoking in other cohort of smokers are needed and the reliability of the information obtained by face to face interview as used in our study should be compared to that obtained from selfadministered questionnaires. 


\section{References}

1. Mathers CD, Loncar D. Projections of global mortality and burden of disease from 2002-2030. PLoS Med 2006;3:e442.

2. Degenhardt L, Chiu WT, Sampson N, et al. Toward a global view of alcohol, tobacco, cannabis, and cocaine use: findings from the WHO World Mental Health Surveys. PLoS Med 2008;5:e141.

3. Adekoya BJ, Adekoya A0, Adepoju FG, Owoeye GFA. Driving under influence among long distance commercial drivers in Ilorin, Nigeria. Int $\mathrm{J}$ Biol Med Res 2011;2:870-3.

4. Aniebue PN, Okonkwo KOB. Prevalence of psychoactive drug use by taxi drivers in Nigeria. J College Med 2008;13:48-52.

5. Lasebikan V0, Ojediran B. Profile of problems and risk factors associated with tobacco consumption among professional drivers in Nigeria. ISRN Public Health 2012;2012:1-6.

6. Patrick DL, Cheadle A, Thompson DC, et al. The validity of self-reported smoking: a review and meta-analysis. Am J Public Health 1994;84:1086-93.

7. Gorber SC, Schofield-Hurwitz S, Hardt J, et al. The accuracy of self-reported smoking: a systematic review of the relationship between self-reported and cotinineassessed smoking status. Nicotine Tob Res 2009;11:12-24

8. Montalto NJ, Wells WO. Validation of selfreported smoking status using saliva cotinine: a rapid semi quantitative dipstick method. Cancer Epidemiol. Biomarkers Prev 2007;16:1858-62.

9. Jarvis MJ, Tunstall-Pedoe H, Feyerabend $\mathrm{C}$, et al. Comparison of tests used to distinguish smokers from non-smokers. Am J Public Health 1987;77:1435-8.

10. Muranaka H, Higashi E, Itani S, Shimizu Y. Evaluation of nicotine, cotinine thiocyanate, carboxyhemoglobin, and expired carbon monoxide as biochemical tobacco smoke uptake parameters. Int Arch Occup Environ Health 1988;60:37-41.

11. Jarvis MJ, Russell MA, Benowitz NL, Feyerabend C. Elimination of cotinine from body fluids: implications for non-invasive measurement of tobacco smoke exposure. Am J Public Health 1988;78:696-8.

12. Studts JL, Ghate SR, Gill JL, et al. Validity of self-reported smoking status among participants in a lung cancer screening trial. cancer epidemiol. Biomarkers Prev 2006;15:1825-8.

13. Watts RR, Langone JJ, Knight GJ, Lewtast J. Cotinine analytical workshop report: consideration of analytical methods for determining cotinine in human body fluids as a measure of passive exposure to tobacco smoke. Environ Health Perspect 1990;84:173-82.

14. Gariti P, Rosenthal DI, Lindell K, et al. Validating a dipstick method for detecting recent smoking. Cancer Epidemiol Biomarkers Prev 2002;11:1123-5.

15. Davison G, Duffy M. Smoking habits of long-term survivors of surgery for lung cancer. Thorax 1982;37:331-3.

16. Heatherton TF, Kozlowski LT, Frecker RC, Fagerstrom K0. The Fagerstrom test for nicotine dependence: a revision of the Fagerstrom tolerance questionnaire. Br J Addict 1991;86:1119-27.

17. Mirza NR, Bright JL. Nicotine-induced enhancements in the five-choice serial reaction time task in rats are strain dependent. Psychopharmacology (Berl) 2001;154:8-12.

18. National Cancer Institute (US). Cigars: health effects and trends. NIH publication; no. 98-4302. Smoking and tobacco control monograph; 9. Available from: http://cancercontrol.cancer.gov/tcrb/monographs/9/ Accessed: June 23, 2012.

19. Stayner L, Bena J, Sasco AJ, et al. Lung cancer risk and work place exposure to environmental tobacco smoke. Am J Public
Health 2007;97:545-51.

20. Britton GR, Brinthaupt J, Stehle JM, James GD. Comparison of self-reported smoking and urinary cotinine levels in a rural pregnant population. J Obstet Gynecol Neonatal Nurs 2004;33:306-11.

21. Lewis SJ, Cherry NM, McL Niven R, et al. Cotinine levels and self-reported smoking status in patients attending a bronchoscopy clinic. Biomarkers 2003;8:218-28.

22. Tsutsumi A, Kagawa J, Yamano Y, et al. Relation between cotinine in the urine and indices based on self-declared smoking habits. Environ Health Prev Med 2002;6:240-7.

23. Vartiainen E, Seppälä T, Lillsunde P, Puska P. Validation of self-reported smoking by serum cotinine measurement in a community-based study. J Epidemiol Community Health 2002;56:167-70.

24. Yeager DS, Krosnick JA. The validity of self-reported nicotine product use in the 2001-2008 National Health and Nutrition Examination Survey. Med Care 2010;48: 1128-32.

25. Man CN, Fathelrahman AI, Harn GL, et al. Correlation between urinary nicotine, cotinine and self-reported smoking status among educated young adults. Environ Toxicol Pharmacol 2009;28:92-6.

26. Ahijevych K, Parsley LA. Smoke constituent exposure and stage of change in black and white women cigarette smokers. Addict Behav 1999;24:115-20.

27. Ahijevych KL, Tyndale RF, Dhatt RK, et al. Factors influencing cotinine half-life during smoking abstinence in African American and Caucasian women. Nicotine Tob Res 2002;4:423-31.

28. Kitagawa K, Kunugita N, Katoh T, et al. The significance of the homozygous CYP2A6 deletion on nicotine metabolism: a new genotyping method of CYP2A6 using a single PCR-RFLP. Biochem Biophys Res Commun 1999;262:146-51. 